\title{
Mental models of high reliability systems
}

\author{
Karl E. Weick \\ School of Business Administration, University of Michigan, Ann Arbor, MI 48109-1234, USA
}

\begin{abstract}
Weick, K.E., 1989. Mental models of high reliability systems. Industrial Crisis Quarterly, 3: 127142.

Reliable performance in complex systems is determined in part by the adequacy with which mental models of the system capture accurately the dimensions of system coupling and system complexity. Failure to register coupling and complexity leads the observer to intervene into an imagined technology that does not exist and to convert opportunities for error into actual errors. To decrease the frequency with which this conversion occurs, people can make their models more complex or the systems they monitor less complex. Neither type of change is as daunting as it may appear, and this is illustrated by an analysis of the mental model and system design associated with the invasion of Grenada.
\end{abstract}

\section{Introduction}

Human reliability is conventionally defined as "the probability that a person (1) correctly performs some system-required activity in a required time period (if time is a limiting factor) and (2) performs no extraneous activity that can degrade the system" (Miller and Swain, 1987, p. 221). The probability of human error is often represented by the ratio of errors made to number of opportunities for error. As Roberts (this issue) has noted, the organizations studied by the Berkeley project are those where the denominator is large, the numerator small, and both numbers represent the potential for catastrophe. High reliability organizations strive to prevent an opportunity for error from becoming an occasion of error, especially when errors can enlarge in incomprehensible ways with inconceivable consequences. To accomplish this goal, high reliability organizations seem to pay more attention to the denominator (prevent op- 
portunities for error) than to the numerator (prevent opportunities for error from becoming occasions of error).

In this essay we take a closer look at the numerator. We argue that opportunities are more likely to be converted into errors when mental models of systems that are both complex and tightly coupled understate these two dimensions and thereby become decoupled from the actual state of the system. Thus, reliability can be improved when mental models represent these two dimensions more adequately or when the actual values on these two dimensions themselves become simpler and looser. Reliability is a joint product of the model and that which is modeled.

The argument will be developed in the following manner. First, we examine the unique constraints that are imposed at the micro level by systems that are tightly coupled and interactively complex (Perrow, 1984), systems that we will call "complex" for convenience. We pay special attention to the fact that these systems contain continuous processes, permanent uncertainty and strong emotions. Second, we argue that continuous processes, permanent uncertainty and strong emotions affect the mental models that operators are able to form of complex systems. Third, we argue that mental models are susceptible to disruption of a kind that is relatively unique to complex systems. We label these disruptions "rendition errors." Fourth, it is argued that reliable performance within a tightly coupled complex system can occur when people work on the very same couplings and complexity that impose strain on their mental models in the first place. We illustrate this solution using the invasion of Grenada as an example.

\section{Context created by complex systems}

To understand more about how individuals, small groups and teams achieve reliable performance, we must first understand the context of constraints and opportunities they face. That context is largely implicit in the other articles in this issue, and three key qualities of that context need to be made explicit. $\mathrm{Nu}$ clear carriers, air traffic control systems and nuclear power generation all utilize technologies characterized by continuous processing that is subject to unexpected interruptions and strong emotions. Continuous processing tightens the couplings among system components. Unexpected interruptions and strong emotions add interactive complexity to these tightly coupled components.

\section{Continuous processes in complex systems}

Continuous processes in complex systems impose their own imperative, the reliability imperative. The shift from efficiency to reliability may constitute the single most important change associated with these systems and their technologies. Reliability is salient in continuous processing because the overriding is- 
sue is to keep the process doing what it does. This means there is a premium on maintaining continuity and the integrity of the process. "Responsibility for assuring operations continuity is more important than responsibility for effort" (Adler, 1986, p. 20).

Reliability has recently been highlighted as an issue of safety in the context of dangerous technologies that have grown more interactively complex (e.g. nuclear power plants). But the issue of reliability is larger than the question of safety. Most of the technologies associated with safety issues are part of a larger group of technologies, all of which involve continuous processes. The problems posed by continuous processing are more visible and consequential in technologies such as nuclear reactors, but the problems are indigenous to all members of this class. Thus, the current concern with issues of reliability is not just a reaction to an increase in the number of dangerous technologies, it is symptomatic of a larger set of unique issues associated with post-industrial technology in general. While efficiency was the hallmark of deterministic industrial era technology, reliability is the hallmark of stochastic, continuous processes associated with the post-industrial technology.

Perrow's (1984) diagnosis that the coincidence of tight coupling and technological complexity has created conditions of interactive complexity and a new family of failures called "normal accidents," may be an early recognition that continuous processing in general presents unique problems that require unique structures.

\section{Unexpected interruptions in complex systems}

Reliability is also threatened in complex systems by unexpected interruptions. Davis and Taylor (1976) suggest that earlier industrial era technologies were deterministic, with clear cause-effect relationships among what is to be done, how it is to be done, and when it is to be done. Newer automated technologies, however, are no longer dominated by determinism. Instead, "people operate in an environment whose 'important events' are randomly occurring and unpredictable" (p. 388).

For example, Buchanan and Bessant (1985) describe the difficulties people had replacing batch production of pigments with computerized process controls, because an understanding of the mechanisms of pigment chemistry was weak (p. 298). Neither the speed of the reactions nor the nature of the side effects were well understood, which meant that manufacturing was "closer to alchemy" than anything else. A world of alchemy is a world of stochastic events. Technical systems which involve dense interaction, systems such as military command and control systems, are continually vulnerable to unexpected interruptions from staff requesting explanations, justifications, revisions, or attention to the fact that they exist (e.g. Metcalf, 1986; Roberts, in press).

While systems have always been threatened by stochastic events, the unique twist in newer technologies is that the uncertainties are permanent rather than 
transient. All technologies surprise operators at first, but as learning develops, surprises decrease. That normal development, however, occurs less often with new technologies, due to their poorly understood processes, continuous revisions of the design of the process, and the fact that implementation of a process is often the means by which the technology itself is designed. Furthermore, with increased dependence on computers, there is the problem that computers often do not give a complete and accurate picture of the state of the process. Even more crucial, when computers do give an accurate picture, that picture can be irrelevant if "operator state identification and control activities gradually become decoupled from actual process state as a function of execution problems or the unexpected" (Woods et al., 1987, p. 1741).

The gradual decoupling of a mental model of a process from the actual steps that occur in that process, allows events to unfold which ramify in their consequences and grow increasingly incomprehensible. Furthermore, the operator's formation of intentions, choice of control activities, and control intervention can create a novel event that is understood neither by the operator nor by the devices for self-control designed into the material technology itself. Since the operator's representation is itself an intact and plausible view, since the decoupling is gradual, and since the immediate consequences of this decoupling are invisible except for dial fluctuations that could be errors, or independent deviations of separate sensors, or a single problem with multiple symptoms, it is not surprising that "mistakes" persist until a "fresh viewpoint enters the situation" (Woods et al., 1987, p. 1745).

These outcomes can potentially occur whenever events interact unnoticed and unmonitored such that a new technical system and set of interactions is created without anyone intending it. It is this sense in which humans can transform a linear simple system into one that is interactively complex.

\section{Strong emotions in complex systems}

The emphasis on cognition up to this point must be balanced by an equally strong recognition that reliable performance occurs in the context of strong emotions.

When people interact with older, more mechanized technologies, they mostly supply energy and guide tools. As they approach their physical limits in these two activities, they do so gradually and visibly. But with newer technologies where people supply regulation and control rather than energy, the work has become more private, more mental, more complex and more subject to human limitations on information processing. Not only have tasks become more difficult with less forewarning that performance will collapse, but organizations have become more dependent on individual performers, even if this dependence is not recognized by appropriate delegation of authority and autonomy. The singular effect of these changes is that they reduce the amount of control people feel they have over their work setting. As predictability and control de- 
crease, people experience higher arousal, more stress and stronger emotions (Karasek, 1979; Sutton and Kahn, 1987). Thus, we suspect that high arousal is a frequent accompaniment of mental work in complex systems.

High arousal has predictable effects on performance. Any task, especially one with stochastic elements, contains several cues which are irrelevant to performance but are noticed anyway. People are distracted by irrelevant cues when arousal is low, and performance may suffer. As arousal increases, these irrelevant cues are ignored and performance improves. Once all irrelevant cues have been ignored then relevant cues begin to be ignored and performance deteriorates (Easterbrook, 1959). Thus, an inverted U relationship between arousal and performance is hypothesized.

The key point for technology is that the breadth of attention varies in response to fluctuations in emotion (see, for example, Weltman et al., 1971). This variation could affect both task conceptions and performance. It could affect task conceptions because people try to make sense of whatever they notice. As the breadth of their attention varies, so too should their descriptions of what they are doing. Thus, task conceptions themselves, not just task performance, should change as arousal changes. Unreliable performance may persist because the operator is performing a different task than observers realize.

The effect of arousal on task conception is illustrated by Barley's (1986) study of medical staff adaptation to CAT scan technology. Technicians at Urban hospital were the object of a steady stream of directives, imperative speech, puzzling countermands, sarcasm and usurped control generated by radiologists. These could easily have raised the level of threat and arousal experienced by technicians. These increases could have narrowed their attention, made complex learning more difficult and actually altered their conception of what kind of task a CAT scanner technology posed for them. These combined effects would slow their learning, which should have further intensified the pressure that radiologists imposed, making further learning by the technologists even more difficult. Since ongoing learning is so much a part of new technologies, anything that obstructs learning, such as arousal, is of considerable importance.

Even though new technologies make greater demands for abstract mental work, we must pay as much attention to the fact that those are demands as to the fact that they are mental. To cope with mental workload is an arousing, emotional experience, which means that mental processes and products will be modified by affect. As attention varies, so too do conceptions of the technology and the effectiveness and reliability of performance.

\section{Mental models of complex systems}

Continuous processing, unexpected interruptions and strong emotions become important at the individual level because they make it difficult for people to comprehend the system. That difficulty is serious because of the unusual 
skill requirements that are associated with reliable performance in complex systems.

A large repertoire of skills must be maintained even though they are used infrequently. People are usually on standby giving special attention to start-up and to anticipating faults that might lead to downtime. The distinction between operations and maintenance is blurred, skills in monitoring and diagnostics are crucial, and people must be committed to do what is necessary on their own initiative and have the autonomy to do so. Humans have assumed the role of "variance absorber, dealing with and counteracting the unexpected" (Davis and Taylor, 1976, pp. 388-389).

To absorb variance, people must first sense the variance. And it is this requirement which highlights the importance of mental models. More and more of the work associated with new technologies has disappeared into machines, which means that managers and operators must rely more heavily on inference, imagination, intuition, problem solving and mental maps, to monitor and understand what is going on out of sight. Buchanan and Bessant (1985) argue that people who work with new technologies have to have a complex understanding of at least four components: " 1 . the process - its layout, sequence of events and interdependencies; 2 . the product - its key characteristics, properties and variability of raw materials; 3 . the equipment - their functions, capabilities and limitations; 4 . the controls - their functions, capabilities and limitations, and the effects of control actions on performance" (p. 303). These four understandings are crucial because the technology is partially self-controlled, and people have to handle the unexpected and provide backup control when automatic control systems fail. People need sufficient understanding of abstract events so that they can intervene at any time and pick up the process or assemble a recovery.

The significance of these demands is that they force people to develop mental models (e.g. Gentner and Stevens, 1983; Rouse and Morris, 1986) of complex systems. A mental model is a representation of a system that explains how defined inputs are transformed into defined outputs by some plausible cascade of causal connections. Carroll and Olson (1988) describe a mental model as "a rich and elaborate structure, reflecting the user's understanding of what the system contains, how it works, and why it works that way. It can be conceived as knowledge about the system sufficient to permit the user to mentally try out actions before choosing one to execute" (p. 51).

Mental models represent a kind of conceptual machine that simulates processes in some actual target machine. The conceptual simulations are usually based on either a metaphor which compares the target system with some other system already known to the user (e.g. a text editor is a typewriter) or a network representation (Carroll and Olson, 1988, p. 52) which captures the state a system can be in and the actions that will transform it to another state. Both representations are helpful in problem solving, but that help is limited because neither representation captures why the system elements are related the way 
they are nor how the components themselves behave. Furthermore, representations in the form of metaphors and networks vary in the degree to which they portray accurately the complexity of the target system and the tightness of its connections.

The importance of mental models for problems of reliability is that the technologies which drive reliable systems are basically dual rather than singular. They involve the material technology of the largely invisible process that is actually unfolding, and the implicit technology of an imagined process that exists in the mind of an individual or team. Complex systems have parallel technologies, a technology in the head and a technology on the floor. Each technology is self-contained. Each is coordinated with the other intermittently rather than continuously. Each corrects the other discontinuously. Each can have a sizeable effect on the other. There are relatively few points at which the mental representation can be checked against and corrected by the actual process. True, there are hundreds of discrete sensors that track fluctuations, but those readings do not convey a direct picture of relationships.

Thus, unlike other technologies that have been studied by organizational theorists, newer technologies exist as much in the head of the operator as they do on the plant floor. This is not to argue that one technology is more important than the other. But it is to argue that cognition and micro-level processes are a key to understanding reliable performance.

\section{Imperfections in mental models}

Traditionally, the errors that produce unreliability have been classified as errors of omission and errors of commission. Miller and Swain (1987) describe the distinction this way: "A person can make an error if he does something incorrectly, fails to do something he should, or fails to do something in time. An error of omission occurs when an operator omits a step in a task, or the entire task. Thus, when a mechanic changes engine oil and puts the new oil in without replacing the oil-pan drain plug, his omission error results in wasted oil and a messy garage floor. An error of commission occurs when an operator does the task, but does it incorrectly. This is a broad category, encompassing selection errors, sequence errors, time errors and qualitative errors. In the oilchange example, if the mechanic chooses the wrong-sized socket for tightening the drain plug, he has made a selection error. If he adds new oil before draining the old oil, he has made an error in sequence. If he cannot finish the job in the allotted time, he has made a time error. If he puts too little torque on the oilpan drain plug, causing it to leak slowly, he has made a qualitative error" ( $p$. 221).

Now suppose that what it means to do something incorrectly is ambiguous because there are multiple criteria and trade-offs. Suppose, further, that incomplete cause-effect knowledge makes it difficult to prescribe procedures and 
makes it easy to prescribe the use of judgement and improvisation. And suppose, finally, that multiple events occur simultaneously, out of sight, and that the pace with which they unfold is variable. The resulting picture approximates complex systems more accurately, and suggests the need to think differently about what an error means under such conditions.

High reliability systems engage in continuous processing, often assisted by less visible electronic processes, under conditions where there is incomplete cause-effect knowledge. Since there is less integration by the machine itself of discrete measures of system performance (one measurement-one dial), there must be more integration of data by human monitors. These human monitors are themselves often tense operators due to the unpredictability of the environment and the uncontrollability of the process. The combination of low predictability and low control obstructs learning, slows responsiveness and underutilizes accumulated experience.

These unique characteristics of high reliability systems create an increased number of opportunities for error because some of them transform the system from a linear system into a complex system (e.g. continuous processing, incomplete cause-effect knowledge, increased demands for integration of data) and some of them transform a loosely coupled system into a tightly coupled system (e.g. continuous processing, centralization, electronic interconnections). This increased number of opportunities for errors is often then converted into an increased number of actual errors when people try to manage the system using representations of the process that are more linear and less tight than the process itself. Consequently, the representations are simpler and slower, which means that interventions into the actual process based on them will be misplaced, mis-timed, and misunderstood because the process state has been misidentified.

This is what we mean by the phrase "rendition error." An error of rendition occurs when a person builds a version (rendition) of the process that fails to match the level of complexity and tightness of coupling that is characteristic of the process. Reliance on the mismatched rendition leads to misidentification of the state of the actual process. This is not to suggest that all cognitive simplification increases the probability of error. Instead, we are suggesting that out of all possible simplifications that can be made when people model a process, misjudging the dimensions of coupling and complexity are the most serious for reliable performance. They are the most serious because these are the two dimensions that, if unmanaged and unmonitored, increase the likelihood of normal accidents. In complex systems, errors of commission and omission still occur, which means the distinction remains viable. However, these two traditional forms occur relative to the rendition and not relative to the actual process itself.

To worry about errors of rendition is not to ignore the material system itself. Material artifacts set sensemaking processes in motion, and sensemaking has no alternative but to deal with technology rather than ignore it. What we are 
trying to emphasize is not that people ignore technology, but rather that the increased mental workload created by stochastic continuous events forces people to impose more of their own interpretations to understand what is occurring.

Interactive complexity is intrinsically difficult to understand, which means that whatever understanding people do arrive at, has a substantially larger proportion of themselves woven into it. People project more of themselves into complex systems of necessity. As they do so, the constraints imposed by bounded rationality limit the number and complexity of the interactions they are able to model. Complex systems could be said to consist of fast technologies and slow minds. People interact as much with their own interpretation of the system which they encode in mental models as they do with the material artifacts themselves. But the artifacts are clearly there throughout the sensemaking process, although exactly what is there is open to a variety of perceptions and conceptions.

Thus, the errors that breach reliable performance are qualitatively different from errors that breach efficient performance. Efficient performance is breached when people stray from guidelines and err in executing an intention. Reliable performance is breached when people misidentify the state of a process and form the wrong intention. Incomplete mental models are an important source of wrong intentions. And underestimates of tight coupling and technical complexity are an important source of incomplete models.

\section{Coping with imperfections in mental models}

To increase the accuracy with which coupling and complexity are represented in mental models, people can do one of two things. They can make their models more complex or the processes they model more simple. Both tactics were used by Metcalf when he commanded the Grenada invasion. A careful examination of some of his tactics suggests how representations are kept coupled with the complex systems they represent.

\section{Command and control in Grenada}

This analysis is focused on the communication technology that Metcalf (1986) constructed to manage military forces during the Grenada rescue operation.

Metcalf's strategy was heavily influenced by his earlier experience with the evacuation of Saigon. Saigon was a politically charged situation in which the on-scene commanders were the object of " 'the six thousand mile screwdriver'

- the minute direction of the day-to-day operations of a field commander by higher and remote authority" (p. 278).

Grenada was also a politically charged situation and raised the same temptations for remote direction. Metcalf described the context this way: "I knew 
the Grenada operation represented tremendous political risks. Because of the risks, particularly in peacetime, there is a natural tendency for higher authority to move down and attempt to control local actions from afar. In this day of instant communications from and to anywhere in the world, combined with the high stakes involved, the local commander has an obligation to communicate information up if he expects to retain control. Higher command authority must always have information, or they will remove control from the local commander" (p. 285).

To retain local control, Metcalf used situation reports, a command voice, explicit advance plans, staff warnings and a party line to enact an environment up the chain of command that told him to do essentially what he wanted to do. "I organized the structure of decision making in a way that, I thought, would maximize the delegation of authority down to me, whereas below me, I delegated 'what' to do to my subordinates and they were responsible for the 'how' " (p. 279).

Metcalf directed his staff to send at least two situation reports upward every hour, whether there was anything to report or not. Not only did these reports keep the National Command Authority informed, but they also kept their staffs busy "reading messages, not telling me what to do" (p. 285). These reports were written in plain English rather than computer format which meant that they could be used (and were used) by a wide variety of audiences to stay abreast of happenings (p. 291). These audiences became dependent on the reports which enabled Metcalf to influence how these audiences defined the situation in Grenada. Because this reporting system was reliable and anticipated queries before they became issues, its reports were taken to be the "true" state of affairs, even when other communication channels into the Pentagon carried information that was at odds with Metcalf's version. Conflicting information is inevitable in "the fog of battle." The problem is, when people with competing interests try to decide which is the "true" version, the fog intensifies, decision making is slowed, opportunities are lost and contradictory directives are issued.

In addition to the frequent situational reports, Metcalf also assigned an experienced operations officer to the one secure phone from Grenada to headquarters in Norfolk (p. 285) and this same person was the command voice during all active combat operations. "The commander in chief and his staff always heard the same voice, a voice they knew and could relate to. The object was to create the impression that, in fact, we were in control and knew what was going on" (p. 285).

Control was also gained through the use of advance planning, alteration of staff job descriptions and public communication. Operational plans for the next day were formulated by 5:00 PM each day so that higher authorities could review Metcalf's intentions in the context of his own estimates of needs and strengths. "Usually they turned my 'intentions' into a directive telling me to do what I told them I was going to do" (p. 285). 
Staff people responsible for briefing their bosses also represented a threat to intentions because they overloaded Metcalf's staff with requests for information that might suggest other lines of action. Most of the information they requested had been provided in previous messages so Metcalf, early in the operation, "informed" the watch officer for the US Commander-in-Chief of the Atlantic Command, at 4:00 AM, to "please get off my back" (p. 292). The unexpected "request" worked and a staff officer was then assigned the task of screening everything sent to Metcalf. "I am told they had an enormous reject bin" (p. 292).

And finally, because of limited communication equipment, the entire Grenada task force was on a party line. "When either my call sign or Admiral McDonald's went out over the circuit, the line was instantly cleared. Captains of the ships huddled around the CIC to listen in, and so this particular party line came to serve a very useful function. It conveyed our intentions to those commanders, without my having to go over them again individually with each captain ... Everyone involved in the mission decision making knew the intended plans and the pressures on local command" (p. 292).

\section{Matching models with systems in Grenada}

Metcalf's experience in Grenada is relevant to our more general interest in reliable performance because he managed a continuous process that was subject to unexpected events (e.g. Cuban strength was seriously underestimated). The system itself generated repeated opportunities for error, both because it was interactively complex (e.g. activities of different armed services had to be coordinated using tourist maps because military maps did not exist for this area ) and because it was tightly coupled (e.g. Rules of Engagement such as "minimize casualties" (p. 282) imposed constraints on the choice of options). What is instructive is the way Metcalf worked within these constraints to make his mental model more complex and tightly coupled while he simultaneously worked to make the system he modeled less complex and more loosely coupled. These joint efforts decrease the chance that his mental model becomes decoupled from the system it is designed to model. As the probability of decoupling decreases, so too does the probability that an opportunity for error will become an actual error.

We look first at the dimension of complexity. Metcalf's earlier experience in Saigon gave him a rough model to start with in structuring his command and control system in Grenada. He had some idea what to expect, but perhaps even more important, he had some idea of how his expectations could be interrupted and micro-managed from afar. The Saigon experience, in short, increased the repertoire of skills and diagnoses Metcalf brought to the situation in Grenada. This increment is crucial because it has been suggested that capability affects perception (Jervis, 1976). People will perceive those events which they believe they can do something about, and will neglect those for which they 
have no response. People with many skills can afford to notice more details because whatever they find, there is a good chance that they can do something about it. Thus a person's repertoire has a significant effect on how much of the situation is noticed. The larger the repertoire, the larger the set of goals among which one can choose, because increased perception is likely to reveal more leverage points in the situation. The point is that Metcalf's experience in Saigon increased the complexity of his mental models which in turn allowed him to simplify the actual system he built in Grenada. Both changes improve the match between the model and that which is modeled on the relevant dimension of complexity.

Other ways in which complexity is managed in Grenada include the use of face-to-face meetings (p. 289). Daft and Lengel (1984) have shown that faceto-face conversations are rich media capable of sensing a greater range of complexity in situations. Thus, the frequent use of rich media improves the match between the complexity of Metcalf's models and the situations those models represent. As both Roberts and Rochlin note (this issue), negotiation, bargaining and talk occur nonstop on nuclear carriers. This can be viewed as a continuing effort to match the complexity of an ongoing system, with the most complex human activity capable of sensing that complexity, face-to-face interaction.

Metcalf's continuing revision of structures (e.g. his use of General Schwarzkopf in a variety of roles such as liaison, deputy, p. 281) can be viewed as a novel solution to a classic problem in organizational structure. When faced with complex environments and non-routine problems, organizations typically choose one of two solutions. They either divide the problem into lots of pieces and coordinate the solutions by means of complex structures or they keep the problems intact and assign them to complex people such as professionals (Scott, 1987, p. 236).

While Metcalf leaned toward the latter solution, he also allowed the interactions among professionals to construct structures which then affected further interactions in the field, with a further effect on structure. Thus, structure is both an independent and dependent variable in Metcalf's design. He relies on complex performances rather than upon complex performers or complex structures. This solution is closer to the newer models of structuration in the organizational literature (e.g. Barley, 1986; Turner, 1988) than to the older models of fixed structures' built-in response to technological imperatives.

Structuration is an elegant way to build a mental model of a complex system. It simultaneously simplifies the system being modeled (interaction creates an interpretable structure) and it complicates the models of the people who are doing the modeling (the structure that is created forces people to pay more attention to one another). Thus the model and that which is modeled match more closely.

Not only are Metcalf's designs sensitive to issues of complexity, they are also sensitive to issues of coupling. Metcalf manages upward in order to "maximize the delegation of authority down to me." Said differently, Metcalf manages in 
order to loosen the coupling between himself and his superiors, but he does so, not by ignoring them, but by demonstrating repeatedly that he is a credible commander (Henshel, 1987). Metcalf also loosens the coupling between himself and his subordinates by telling them what to do but allowing them to determine how they will do it.

What is interesting about these efforts is that they loosen the couplings among system parts, but in doing so, they also reduce complexity. They reduce complexity because they decrease the likelihood that events can ramify widely, in incomprehensible ways, with unanticipated consequences. A system with looser couplings is often also a system that is less complex. While complexity and coupling are typically viewed as orthogonal dimensions, the arguments raised here suggest that changes in one dimension can affect the other. Metcalf constructed a loosely coupled system that he knew and could comprehend, but the system was also less complex because of the prior experience he had had under related conditions.

Efforts to loosen couplings are also visible in Metcalf's insistence that his intentions for tomorrow's actions be in the hands of his superiors by 5:00 PM the preceding day. This builds in time to negotiate should the superiors prefer a different strategy than the one proposed. This tactic loosens the coupling between today and tomorrow, a coupling that is normally tight when operations are continuous. In a tightly coupled world, what I will do at 5:00 AM is often determined by what I did at 4:58 AM and was told to do by my superiors at 4:59 AM. In a loosely coupled world, what I will do at 5:00 AM can be anything that I am able to negotiate between 5:00 PM the previous evening and 5:00 AM the next morning.

Metcalf's decisions within the context of the Rules of Engagement (p. 281) provide an interesting example of simultaneous loose-tight coupling. Metcalf repeatedly turns to the three Rules of Engagement for guidance, invokes them consistently as constraints on his own decisions, but retains discretion over everything else. What is interesting about this is that it appears to be a good example of simultaneous centralization and decentralization, a structural combination which is crucial for reliable performance yet rare in ongoing organizations (Weick, 1987, pp. 124-126). The premises for Metcalf's decision are centralized, but what he does within these broad constraints is left to his discretion. Thus, both the efficiency of centralization and the effectiveness of decentralization are possible within this structure. Most important, Metcalf's use of the Rules of Engagement reduces both tight coupling and interactive complexity. Tight coupling is reduced because premises are translated into decisions that are responsive to local conditions. Complexity is reduced because decisions that are locally responsive create a system that is easier to comprehend, easier to model and easier to keep track of.

While we could elaborate the Grenada example even further, the point we wish to make is that systems characterized by tight coupling and interactive complexity can be managed toward levels of high reliability, if mental models 
are made more complex and if systems are made less complex. Models are made more complex by such devices as diverse experience, pooling of experience among people, imagination, narrative thinking and training in cognitive integration. Systems are made less complex through such devices as delegation of authority, proactive management upward, placing people rather than machines in control loops and using talk to hold the system together.

\section{Conclusion}

What we have tried to show is that the micro-structure of reliable performance has a significant cognitive component, due in part to the nature of the systems in which reliability is a salient issue. What is striking about these systems is that their continuous processes are less visible, sensible and comprehensible, yet their reliability is heavily dependent on human interventions that presume high visibility, sensibility and comprehensibility. Operators manage this discrepancy by creating an imagined system. The adequacy of this representation as well as its ability to keep up with the actual system determine reliable performance.

There are several action implications of this perspective. First, mental models of complex systems should be made explicit so that they can be modified. Carroll and Olson (1988), for example, suggest that this can be done if the trainer provides the user with metaphors before the system is studied in order to enrich the imagery in the mental model the user constructs. The trainer can also encourage the user to generate metaphors that are personally meaningful, at each stage in the instruction. In either case, imagery, representation and narrative thinking are encouraged rather than discouraged. Reliable performance is not necessarily dependent on a literal duplication of the system model. That is impossible, but all approximations are not equally useful. Those representations which preserve interactive complexity (e.g. the representation portrays causal interactions that could produce vicious circles; Weick, 1979, Chapter 3 ) and those representations that preserve tight coupling (e.g. the representation portrays swift, large causal effects) are more likely to be useful.

Second, system design should not be treated as a given. To do so is to avoid testing the possibility that small material changes may have large effects (Weick, 1984). What is notable about Metcalf's efforts in Grenada is that they involve close attention to small details that have large effects. People have some control over some aspects of any complex situation. Even though the control appears to be small, that does not mean that it is insignificant.

By definition, systems that are high in interactive complexity are systems in which competence can be multiplied. A 5:00 meeting, a message every half hour and a familiar voice, all seemingly trivial, can set in motion large changes that improve comprehension, manageability and reliable performance. Furthermore, human interventions can also slow a set of changes that are already 
in motion. Metcalf dampens the intrusiveness of staff requests, slows the pace of invasion and has what amounts to several simultaneous conversations over the "party line," all of which enact a system that he can model more accurately with the models that have worked for him in the past.

Third, provisions must be made to acknowledge and accept the emotional intensity of life in complex systems. The mere fact of acknowledgement rather than denial may itself have a calming influence and improve performance. What frightens people are seemingly inappropriate emotions that call into question their grasp of the situation. Situations with no meaning whatsoever are more troublesome and arousing than are situations where the meaning is clear, even if it is unpleasant. Furthermore, things as diverse as social support, user-friendly machine interfaces and counseling to assist with problems away from work, all contribute to reliable performance because they all address the fact that stress is additive. To attack arousal on several fronts is to reduce the extent to which it edits crucial material out of mental models.

Finally, complex, tightly coupled systems impose more demands than any one person can handle. Metcalf, despite all of his expertise, wisdom and intuition, did not manage Grenada singlehandedly. Instead, there was an interdependent team of diverse, credible, trusting people able to envision and act on a collective mental model that was more complex than any one of them could articulate. Collective requisite variety is a prerequisite for reliable performance in complex systems. When the problem is one of adequate representation of invisible events on a continuous basis, there are no great operators, only great operations. There may be many charismatic leaders, but there are few charismatic modelers. Charisma, in complex systems, is a social accomplishment that involves a shared mental model.

\section{References}

Adler, P., 1986. New technologies, new skills. California Management Review, 29: 9-28.

Barley, S., 1986. Technology as an occasion for structuring: evidence from observations of CAT scanners and the social order of radiology departments. Administrative Science Quarterly, 31: 78-108.

Buchanan, D.A. and Bessant, J., 1985. Failure, uncertainty, and control: the role of operators in a computer integrated production system. Journal of Management Studies, 22: 292-308.

Carroll, J.M. and Olson, J.R., 1988. Mental models in human-computer interaction. In: M. Helander (Editor), Handbook of Human-Computer Interaction. North-Holland, New York: $45-65$.

Daft, R.L. and Lengel, R.H., 1984. Information richness: a new approach to manager information processing and organization design. In: B. Staw and L.L. Cummings (Editors), Research in Organizational Behavior (Vol. 6). JAI, Greenwich, CT: 191-233.

Davis, L.E. and Taylor, J.C., 1976. Technology, organization, and job structure. In: R. Dubin (Editor), Handbook of Work Organization. Rand-McNally, Chicago, IL: 379-419.

Easterbrook, J.A., 1959. The effect of emotion on cue utilization and the organization of behavior. Psychological Review, 66: 183-201. 
Gentner, D. and Stevens, A., 1983. Mental Models. Erlbaum, Hillsdale, NJ.

Henshel, R., 1987. Credibility and confidence feedback loops in social prediction. Paper presented at the Plenary Session of the VII International Congress of Cybernetics and Systems, University of London.

Jervis, R., 1976. Perception and Misperception in International Politics. Princeton University Press, Princeton, NJ.

Karasek, R.A., 1979. Job demands, job decision latitude and mental strain: implications for job design. Administrative Science Quarterly, 24: 285-308.

Metcalf, J., III, 1986. Decision making and the Grenada rescue operation. In: J.G. March and R. Weissinger-Baylon (Editors), Ambiguity and Command. Pitman, Marshfield, MA: 277297.

Miller, D.W. and Swain, A.D., 1987. Human error and human reliability. In: G. Salvendy (Editor), Handbook of Human Factors. Wiley, New York: 219-250.

Perrow, C., 1984. Normal Accidents. Basic Books, New York.

Roberts, K.H., 1989. New challenges in organizational research: high reliability organizations. Industrial Crisis Quarterly, 3: 111-125 (this issue).

Roberts, K.H. (in press). Bishop Rock dead ahead: the grounding of the USS Enterprise. Naval Institute Proceedings.

Rochlin, G.I., 1989. Informal organizational networking as a crisis-avoidance strategy: US naval flight operations as a case study. Industrial Crisis Quarterly, 3: 159-176 (this issue).

Rouse, W.B. and Morris, N.M., 1986. On looking into the black box: prospects and limits in the search for mental models. Psychological Bulletin, 100: 349-363.

Scott, W.R., 1987. Organizations: rational, natural, and open systems (2nd ed.). Prentice-Hall, Englewood Cliffs, NJ.

Sutton, R.I. and Kahn, R.L., 1987. Prediction, understanding, and control as antidotes to organizational stress. In: J. Lorsch (Editor), Handbook of Organizational Behavior. PrenticeHall, Englewood Cliffs, NJ: 272-285.

Turner, J.H., 1988. A Theory of Social Interaction. Stanford University Press, Stanford, CA.

Weick, K.E., 1979. The Social Psychology of Organizing. Addison-Wesley, Reading, MA.

Weick, K.E., 1984. Small wins: redefining the scale of social problems. American Psychologist, 39: 40-49.

Weick, K.E., 1987. Organizational culture as a source of high reliability. California Management Review, 29: 112-127.

Weltman, G., Smith, J.E. and Egstrom, G.H., 1971. Perceptual narrowing during simulated pressure-chamber exposure. Human Factors, 13: 99-107.

Woods, D.P., O'Brien, J.F. and Hanes, L.F., 1987. Human factors challenges in process control: the case of nuclear power plants. In: G. Salvendy (Editor), Handbook of Human Factors. Wiley, New York: 1724-1770. 